\title{
Cortical barrel lesions impair whisker-CS trace eyeblink conditioning
}

\author{
Roberto Galvez, ${ }^{1}$ Aldis P. Weible, and John F. Disterhoft \\ Department of Physiology, Northwestern University Feinberg School of Medicine, Chicago, Illinois 60611, USA
}

\begin{abstract}
Whisker deflection is an effective conditioned stimulus (CS) for trace eyeblink conditioning that has been shown to induce a learning-specific expansion of whisker-related cortical barrels, suggesting that memory storage for an aspect of the trace association resides in barrel cortex. To examine the role of the barrel cortex in acquisition and retrieval of trace eyeblink associations, the barrel cortex was lesioned either prior to (acquisition group) or following (retention group) trace conditioning. The acquisition lesion group was unable to acquire the trace conditioned response, suggesting that the whisker barrel cortex is vital for learning trace eyeblink conditioning with whisker deflection as the CS. The retention lesion group exhibited a significant reduction in expression of the previously acquired conditioned response, suggesting that an aspect of the trace association may reside in barrel cortex. These results demonstrate that the barrel cortex is important for both acquisition and retention of whisker trace eyeblink conditioning. Furthermore, these results, along with prior anatomical whisker barrel analyses suggest that the barrel cortex is a site for long-term storage of whisker trace eyeblink associations.
\end{abstract}

Pavlovian conditioning has been used as a tool to study the various aspects of memory acquisition and retrieval for over a century. In Pavlovian conditioning, a neutral conditioned stimulus (CS) is paired with a salient unconditioned stimulus (US) that elicits an unconditioned response (UR). After repeated CS-US pairings, the animal begins to exhibit a conditioned response (CR) that precedes US onset. In delay conditioning, the CS and US are not temporally separated. This form of conditioning is forebrain independent, in that decerebration, decortication, or hippocampectomy does not impair acquisition (Theios and Brelsford Jr. 1966; Norman et al. 1977; Oakley and Russell 1977; Mauk and Thompson 1987). Rather, delay conditioning is dependent upon brainstem and cerebellar processing (Clark et al. 1984; Mauk and Thompson 1987). In trace conditioning, the CS and US are temporally separated by a stimulus free trace interval. This form of conditioning, in addition to being dependent upon the same brainstem and cerebellar processing as in delay conditioning, is also dependent upon midbrain structures such as the medial dorsal thalamus (Powell and Churchwell 2002) and upon forebrain regions such as the hippocampus (Solomon et al. 1986; Moyer Jr. et al. 1990; Kim et al. 1995; McGlinchey-Berroth et al. 1997; Clark and Squire 1998; Weiss et al. 1999; Takehara et al. 2002; Tseng et al. 2004), medial prefrontal cortex (McLaughlin et al. 2002; Takehara et al. 2003; Frankland et al. 2006), and anterior cingulate cortex (Kronforst-Collins and Disterhoft 1998; Weible et al. 2000; Han et al. 2003). For reviews of the circuitry involved in trace eyeblink conditioning, see Berger and Bassett (1992), Weiss and Disterhoft (1996), and Weiss et al. (2006).

Analyses of hippocampal involvement in trace conditioning have demonstrated that lesioning the hippocampus prior to, or $1 \mathrm{~d}$ after learning, impairs an animal's ability to form trace associations (Solomon et al. 1986; Moyer Jr. et al. 1990; Kim et al. 1995; McGlinchey-Berroth et al. 1997; Clark and Squire 1998; Weiss et al. 1999; Takehara et al. 2002; Tseng et al. 2004). However, hippocampal lesions made $30 \mathrm{~d}$ after training do not impair an animal's ability to exhibit appropriately timed CRs (Kim et al. 1995; Takehara et al. 2002), suggesting that long-term storage for

${ }^{1}$ Corresponding author.

E-mail r-galvez@northwestern.edu; fax (312) 503-5101.

Article is online at http://www.learnmem.org/cgi/doi/10.1101//m.418407. trace associations occurs elsewhere in the brain. Various behavioral paradigms, such as frequency discrimination training (Disterhoft and Stuart 1976; Kitzes et al. 1978; Diamond and Weinberger 1986, 1989; Edeline et al. 1993; Recanzone et al. 1993; Rutkowski and Weinberger 2005) and tactile discrimination training (Jenkins et al. 1990; Recanzone et al. 1992; Krupa et al. 2004), induce plasticity in the neocortex. These and similar studies, have led to theoretical models (Eichenbaum et al. 1992; Squire et al. 2004) suggesting that one of the most likely locations for long-term storage of trace associations is the neocortex.

Recent work from our laboratory has focused on utilizing whisker deflection as the CS to facilitate examining long-term storage of trace associations. These studies have demonstrated that whisker deflection is an effective CS for both delay (Das et al. 2001) and trace eyeblink conditioning (Galvez et al. 2006; LealCampanario et al. 2006). Furthermore, we have recently demonstrated that using whisker deflection as the CS induces a learningspecific expansion of whisker-related cortical barrels compared with pseudo-conditioned animals, demonstrating that the expansion was not simply due to use (Galvez et al. 2006), thus suggesting that memory storage for an aspect of the trace association occurs in barrel cortex.

We report an analysis of the role of the barrel cortex in acquisition and retrieval of trace eyeblink associations carried out by lesioning the barrel cortex either prior to (acquisition group) or following (retention group) trace conditioning (Fig. 1). These analyses demonstrated that the barrel cortex (1) is required for initial acquisition of trace associations and (2) plays a role in retention/expression of previously acquired CRs. Note, both acquisition and retention lesion groups were able to learn delay conditioning, suggesting that the inability to learn or exhibit already learned trace associations was not due to an inability to perceive the CS. These results suggest that the barrel cortex is involved in both the initial acquisition and subsequent retrieval of the trace association.

\section{Results}

Performance of acquisition lesion pseudo-conditioned animals did not significantly differ from acquisition sham pseudoconditioned animals for any of the analyses performed. There- 
A Acquisition groups

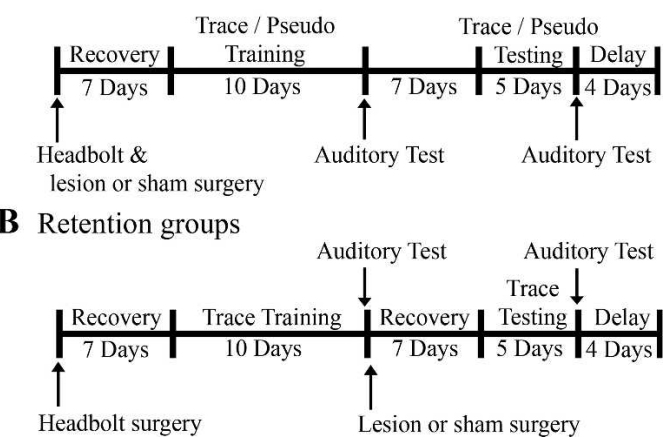

Figure 1. Schematic of experimental design.

fore the data from these groups were combined and will be referred to as pseudo-conditioned from here on.

Data from animals receiving ibotenic acid infusions were included in the appropriate lesion groups only if all or all but one of the B-row barrels were absent in cytochrome oxidase (CO)reacted sections (Fig. 2). Four of the ibotenic acid-infused animals had partial (more than one spared barrel) or complete sparing of B-row cortical barrels. Performance of these animals was not significantly different from, and was thus included in, the appropriate sham groups.

\section{Acquisition group}

The barrel cortex was lesioned prior to trace eyeblink conditioning to assess the role of the barrel cortex in acquisition of the trace association. Lesioned animals did not exhibit a significant change in the percentage of adaptive CRs across days, did not significantly differ in the percentage of adaptive CRs from pseudo-conditioned animals, and had significantly fewer adaptive CRs than did acquisition sham animals during trace training $\left(F_{(1,11)}=11.25 ; P<0.05\right)$ and subsequent trace testing $\left(F_{(1,11)}=77.19 ; P<0.05\right)$ phases of the experiment (Fig. 3A). Acquisition sham animals exhibited a significant increase in the percentage of adaptive CRs across days $\left(F_{(9,72)}=10.20 ; P<0.05\right)$, demonstrating acquisition of the trace association (Fig. 3A). Analyses of UR peak height, peak time, duration, and area during the first $4 \mathrm{~d}$ of trace training demonstrated no significant differences between acquisition sham and lesion animals, as expected due to UR dependence on brain stem processing, suggesting that lesioning of the whisker barrel cortex did not hinder the ability to blink in response to the corneal air-puff. Acquisition lesion animals did exhibit a mean UR onset occurring $9 \pm 3$ msec earlier than acquisition sham animals $\left(F_{(1,51)}=16.59 ; P<0.05\right)$. This difference may reflect a minor modification of brainstem US processing resulting from a disruption of cortical processing but would not in itself explain the dramatically reduced CR percentage exhibited by acquisition lesion animals.

\section{Retention group}

For the retention lesion animals, the barrel cortex was lesioned after trace eyeblink conditioning to assess the role of the barrel cortex in retention of the trace association. During trace testing, the retention lesion animals exhibited a significant reduction in the percentage of adaptive CRs compared with retention sham animals $\left(F_{(1,64)}=15.34 ; P<0.05\right)$ (Fig. 3B). Retention sham and lesion animals both exhibited a significant increase in percentage of adaptive CRs during the trace training phase of the experiment $\left(F_{(9,36)}=10.67 ; P<0.05\right)$, demonstrating comparable acquisition of the trace association (Fig. 3B). Analyses of retention lesion animal UR properties (onset, peak height, peak time, duration, and area) on day one of trace testing did not significantly differ from sham animals, suggesting that the drop in percentage of adaptive CRs was not due to a lesion-induced impairment in US perception or UR performance.

Post hoc analysis of the retention lesion group's performance across the trace testing days demonstrated that unlike the retention sham animals, lesioned animals exhibited a significant increase in the percentage of CRs across days $\left(F_{(4,40)}=2.91\right.$; $P<0.05$ ) (Fig. 3B), suggesting reacquisition of the trace association. To determine whether retention lesion animals dropped in performance to pseudo-conditioning levels on the first day of trace testing, a within-subject post hoc analysis of the percentage of CRs on the first 10 trials on day one of trace testing was conducted. This analysis revealed that retention lesion animals were significantly different from pseudo-conditioned animals within the first 10 trials of day one of trace testing $\left(F_{(1,15)}=10.45\right.$; $P<0.05)$, demonstrating that CR performance by retention lesion animals did not drop to pseudo-conditioning levels on day one of trace testing.

Further analysis of the lesion group's performance during trace testing demonstrated an elevated number of nonadaptive CRs (blinks that returned to pre-CS baseline prior to US onset) in retention lesion $($ mean $=19.4 \pm 2.4 \%)$ compared with retention sham $($ mean $=11.0 \pm 2.1 \%)$ animals $\left(F_{(1,72)}=5.83 ; P<0.05\right)$ (Fig. 4). A within-subject post hoc analysis of adaptive versus nonadaptive CR properties for retention lesion animals during trace testing demonstrated that lesioning the barrel cortex did not significantly affect CR onset, suggesting that the lesioned animals were able to perceive the CS without cortical barrel involvement. However, the fact that the nonadaptive CRs were significantly smaller (peak height $F_{(1,44)}=56.45 ; P<0.05$ ) and shorter (duration $F_{(1,44)}=32.77 ; P<0.05$; area $F_{(1,44)}=10.46$; $P<0.05$ ) and peaked earlier (peak time $F_{(1,44)}=61.28 ; P<0.05$ ) compared with adaptive CRs suggests that lesioning barrel cortex impaired CR timing (Table 1; Fig. 4). Increased occurrence of nonadaptive CRs and no significant difference in adaptive and nonadaptive CR onset by lesioned animals suggests that the knowledge of the association was retained; however, these data along with the observed differences in CR properties strongly
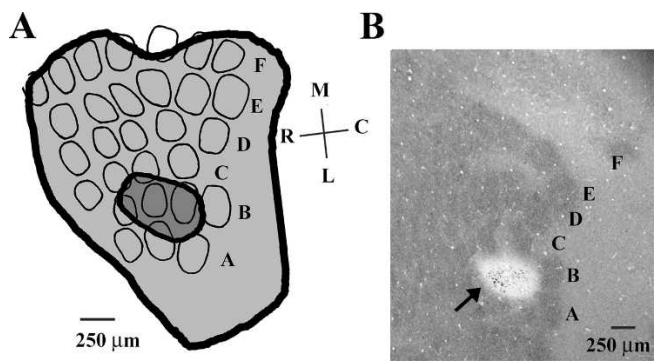

Figure 2. Illustration of somatosensory cortical barrel lesion size. (A) Smallest (dark gray) and largest (light gray) lesion in layer IV of the somatosensory barrel cortex included in the appropriate lesion groups. Cortical barrels from a representative tangential section from a sham animal were delineated for illustrative purposes. Note that the largest lesion did not extend significantly beyond the whisker barrel field in layer IV. The ventral spread of the largest lesion also did not extend all the way through layer VI of the neocortex. $M$ indicates medial; L, lateral; R, rostral; and C, caudal. Letters A-F delineate barrel rows. (B) Cyochrome oxidasestained tangential section through layer IV of barrel cortex with a lesion (arrow) in B row. Note that the white spot in B2-B4 is not a physical hole in the barrel cortex. Brain regions lesioned with ibotenic acid are not as metabolically active as nonlesioned brain regions and thus do not stain as efficiently with cytochrome oxidase (a marker of metabolic activity). Letters A-F delineate barrel rows. 
A Acquisition groups

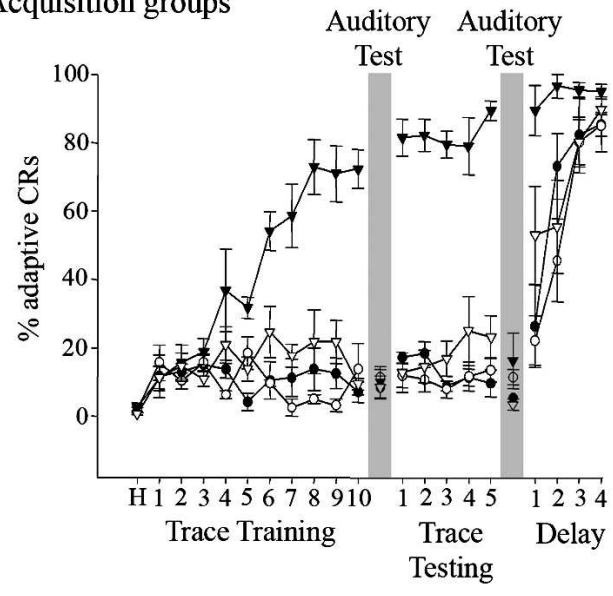

B Retention groups

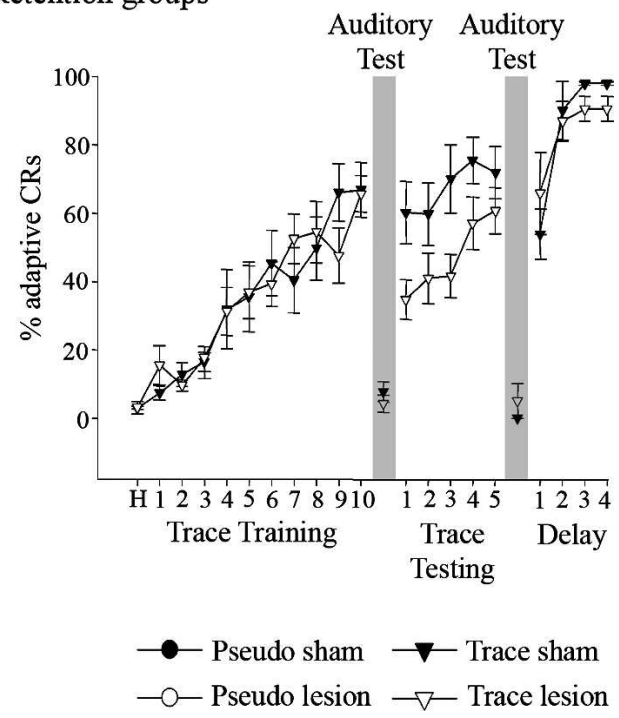

Figure 3. Barrel cortical lesions hinder acquisition and performance of trace eyeblink conditioning. (A) Acquisition groups' performance during trace training, trace testing, and delay conditioning sessions. Note that unlike trace sham animals (trace sham; filled triangle), barrel cortical lesion animals (trace lesion; open triangle) did not learn the trace association and performed comparable to pseudo-condition sham (pseudo sham; filled circles) and lesion (pseudo lesion; open circles) animals. (B) Retention lesion (trace lesion; open triangles) and sham (trace sham; filled triangles) group performances during trace training, trace testing, and delay conditioning sessions. Both groups learned the association during trace training. A drop in performance was observed after lesioning the barrel cortex during trace testing for trace lesion animals. All animals learned delay conditioning. Days 11 and 17 were auditory test days consisting of 20 stimulator detached trials (see Behavioral Training). Auditory test day performances for all animals were at or below pseudoconditioned levels. Error bars, SEM.

suggest that timing or signaling of the learned association was disrupted.

Complete removal of the cerebral cortex has been shown to facilitate subsequent acquisition of reversal conditioning and conditioned inhibition using a delay paradigm (Oakley and Russell 1972, 1975; Moore et al. 1980), a result believed to indicate an inhibitory role for the cerebral cortex in Pavlovian conditioning. Thus, one possibility could be that retention lesions of barrel cortex disrupted neocortical inhibition that then contributed to the increase in nonadaptive CRs. The previous studies also found that decorticate animals failed to exhibit learning-induced changes in CR onset (Oakley and Russell 1972, 1975; Moore et al. 1980); however, in the present study, we observed no significant difference in adaptive versus nonadaptive CR onset, suggesting a different mechanism in trace conditioning.

\section{Auditory test}

All animals received an auditory test after the trace training and trace testing phases of the experiment (Fig. 1, gray boxes; see Behavioral Training) to determine if they were using auditory cues to learn the association. During the auditory test, the piezoelectric strip was adjacent, but not attached, to the animal's whiskers. Animals exhibited a significant reduction in the percentage of adaptive CRs compared with performance on the previous day for acquisition and retention sham animals $\left(F_{(1,54)}=282.50\right.$; $P<0.05$ ) (Fig. 3). Furthermore, performance during the auditory test for all of the groups did not significantly differ from pseudoconditioned animals on the pervious day, suggesting that auditory cues did not contribute to performance of the task.

Delay

All rabbits were trained for an additional $4 \mathrm{~d}$ with a delay conditioning paradigm upon completion of the trace testing phase of the experiment (Fig. 1). Acquisition of delay conditioning is dependent upon brainstem and cerebellum circuitry (Theios and Brelsford Jr. 1966; Norman et al. 1977; Oakley and Russell 1977; Mauk and Thompson 1987), while trace conditioning is additionally dependent upon forebrain circuitry, as discussed by Berger and Bassett (1992), Weiss and Disterhoft (1996), and Weiss et al. (2006). Failure to learn delay conditioning (achieve a minimum of $80 \%$ adaptive CRs within a block of 10 consecutive trials) would be indicative of a more profound disruption of the basic neural circuitry that would preclude acquisition of the more complex trace CR, and would therefore result in exclusion from the experiment. All lesion and sham animals acquired delay eyeblink conditioning normally (Fig. 3).

\section{Discussion}

Previous work has demonstrated that whisker deflection can be used as a CS for trace conditioning (Galvez et al. 2006; LealCampanario et al. 2006). This form of conditioning also induces learning- and whisker-specific changes in primary somatosensory barrel cortex compared with pseudo-conditioned animals, indicating that the expansion was not simply due to use (Galvez et al. 2006), suggesting that the barrel cortex could be a site for long-
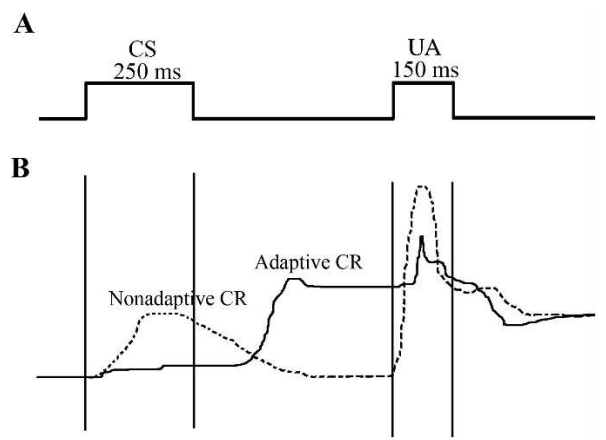

Figure 4. Illustration of adaptive and nonadaptive conditioned responses for retention lesion animals during trace testing. $(A)$ Schematic of the trace conditioning paradigm used. CS indicates conditioned stimulus; US, unconditioned stimulus. (B) Example of an adaptive and nonadaptive conditioned response (CR) on day one of trace testing exhibited by a retention lesion animal. Upward deflection represents a sweep of the nictitating membrane across the cornea.

\section{Learning \& Memory}


Table 1. Conditioned response (CR) properties for adaptive and nonadaptive blinks for retention lesion animals during trace testing

\begin{tabular}{lcc}
\hline & Adaptive CRs & Nonadaptive CRs \\
\hline Onset (msec) & $196 \pm 16$ & $202 \pm 17$ \\
Peak height $(\mathrm{mV}) \mathrm{a}^{\mathrm{a}}$ & $0.54 \pm 0.05$ & $0.28 \pm 0.03$ \\
Peak time $(\mathrm{msec})^{\mathrm{a}}$ & $418 \pm 25$ & $284 \pm 18$ \\
Duration (msec) $^{\mathrm{a}}$ & $503 \pm 18$ & $240 \pm 11$ \\
${\text { Area }\left(\mathrm{mV} \mathrm{N}^{*} \mathrm{msec}\right)^{\mathrm{a}}}$ & $135 \pm 12$ & $40 \pm 5$ \\
\hline
\end{tabular}

Retention lesion animals did not exhibit a significant difference in adaptive vs. nonadaptive CR onset during trace testing. However, nonadaptive CRs were significantly smaller and shorter and peaked earlier compared with adaptive CRs. Values represent means for all $5 \mathrm{~d}$ of trace testing \pm SEM. Time $0=$ CS onset.

a $P<0.05$

term storage of an aspect of the trace association. To examine the role of the barrel cortex in acquisition and retrieval of the trace eyeblink association, the barrel cortex was lesioned prior to (acquisition group) or following (retention group) trace conditioning. The acquisition lesion group was unable to learn the trace association (Fig. 3A), suggesting that the barrel cortex is critically involved in forming trace associations. The retention lesion group exhibited a significant reduction in the percentage of adaptive CRs (Fig. 3B), suggesting that an aspect of the memory for the trace association is stored in barrel cortex. Note this does not necessarily suggest that barrel cortex is a site of convergence for the CS and US, a possibility that seems unlikely given the input to this cortical region. Rather, the learning-specific change in this primary sensory neocortical region more likely reflects an enhancement in the response to the vibrissae CS input as this input gains increased behavioral significance during learning. Such an enhancement has been reported in other sensory regions after various types of learning (Disterhoft and Stuart 1976; Kitzes et al. 1978; Kraus and Disterhoft 1981; Diamond and Weinberger 1986, 1989; Jenkins et al. 1990; Recanzone et al. 1992, 1993; Edeline et al. 1993; Darian-Smith and Gilbert, 1994, 1995; Schoups et al. 2001; Ghose et al. 2002; Krupa et al. 2004; Rutkowski and Weinberger 2005). Since both the lesion and sham animals were able to learn delay conditioning, barrel cortical lesions did not impair the animals' ability to sense and respond to the CS. By lesioning the barrel cortex, we only removed neocortical involvement in processing the CS.

It is well established that acquisition of trace associations requires hippocampal processing. Hippocampal lesions performed prior to conditioning completely abolish an animal's ability to form trace associations (Solomon et al. 1986; Moyer Jr. et al. 1990; Kim et al. 1995; McGlinchey-Berroth et al. 1997; Clark and Squire 1998; Weiss et al. 1999; Takehara et al. 2002; Tseng et al. 2004). Given known neocortical projections to hippocampus via entorhinal cortex, barrel cortical lesions may have prevented primary input for the CS from entering the hippocampus. In addition to cortical input, the hippocampus also receives input from the thalamus (Van der Werf et al. 2002; McKenna and Vertes 2004). However, given the inability of acquisition lesion animals to form the trace association, it would seem that these projections are not sufficient to mediate the initial acquisition of the trace association.

As the trace association is consolidated, the hippocampus becomes less engaged while, it is hypothesized, the neocortex permanently stores the trace association (Eichenbaum et al. 1992; Squire et al. 2004). Our retention lesion group exhibited significantly more nonadaptive CRs than did sham controls (Fig. 4 ), suggesting that barrel cortical lesions compromised their ability to exhibit appropriately timed CRs. These observations, along with our prior analysis of conditioning-induced expansion of trained cortical barrels, further suggest that barrel cortex plays a role in storing the memory for, or an aspect of, the timing for the CR.

Barrel cortical lesions in the acquisition group blocked the animals' ability to acquire the trace conditioned response. In contrast, the retention lesion group, following an initial drop in percentage of adaptive CRs, was able to reacquire the trace association in subsequent trace testing sessions, suggesting that another brain region was able to compensate for the missing cortical barrels if the association had been previously learned. A likely structure, ideally positioned to compensate for the missing cortex, would be the thalamus. Tactile information from individual whisker deflections is sent as a topographically organized map of the whisker pad on the face from thalamic barreloids to somatosensory barrel cortex (Woolsey and Van der Loos 1970). Furthermore, studies have demonstrated that not only can the thalamus undergo plastic changes (Jensen and Killackey 1987; Catalano et al. 1995; Krupa et al. 1999) but thalamic plasticity may be regulated by feedback projections from somatosensory cortex (Krupa et al. 1999). This required somatosensory-thalamic feedback for induction of thalamic plasticity (Krupa et al. 1999) could explain why thalamic involvement alone was insufficient to mediate learning in the acquisition lesion group.

In addition to possible thalamic plasticity, a conditioninginduced neuronal network for the long-term storage of the trace association could also account for the retention lesion group's ability to recover during trace testing. As an animal learns a trace association, it is believed that the memory for the association is slowly stored in the neocortex (Eichenbaum et al. 1992; Squire et al. 2004). However, the exact time course for this event is unknown. Studies of single neuron activity during trace conditioning have demonstrated that hippocampal neurons exhibit a robust learning-related pattern of activity during initial acquisition of the trace association that decreases over subsequent days of training (McEchron and Disterhoft 1997; McEchron et al. 2001; Weible et al. 2006). However, by the end of training, hippocampal neurons continue to exhibit learning-related patterns of neuronal activity, although not as robustly as during initial acquisition (McEchron and Disterhoft 1997; McEchron et al. 2001; Weible et al. 2006). This persistence of learning-related hippocampal activity could suggest that the memory for the trace association is still in a transitional phase from the hippocampus to long-term storage in the neocortex (McLaughlin et al. 2002; Powell and Churchwell 2002; Takehara et al. 2002, 2003; Frankland et al. 2006). By lesioning the barrel cortex at the end of the trace training phase of the experiment and thus removing CS-related input to the entorhinal/hippocampal circuit (retention lesion group), we may have removed hippocampal involvement in contributing to the trace association, leaving an immature memory network in the neocortex to take on the load of the association. Since the initial neuronal changes mediating the trace association would have already been established in the neocortex, additional days of training would strengthen these cortical connections and thus improve performance as was observed.

Although this is a plausible hypothesis, we must also account for our observed increase in the frequency of nonadaptive CRs in retention lesion animals during trace testing. One way to account for this observation, along with the previously observed conditioning-induced whisker-specific expansion of CO-stained cortical barrels (Galvez et al. 2006), is for an aspect of the trace association to be stored in barrel cortex. If barrel cortex was only a passive structure providing input to the hippocampal system, there would be no reason for it to undergo the energy taxing process of expanding the trained cortical barrels, as exhibited by increased CO-stained cortical barrel size (Galvez et al. 2006), or 
for the animal to exhibit a loss in ability to exhibit appropriately timed blinks after barrel cortical lesions. Thus during the later phase of trace conditioning, as the association is being stored in the neocortex, according to both this and the previous hypothesis, the barrel cortex provides a site of storage for an aspect of the trace association.

The retention lesion group's ability to recover during trace testing could also involve recruitment of secondary somatosensory cortex or the contralateral somatosensory cortex. Studies examining performance in a T-maze using whisker stimulation to signal the appropriate response have also demonstrated reacquisition following post-training barrel cortical lesions (Hurwitz et al. 1990, 1991; Pazos et al. 1995). These studies demonstrated that rats were able to reacquire the task following both unilateral and bilateral lesions of both primary and secondary somatosensory cortex (Pazos et al. 1995), suggesting that the reacquisition was not due to recruitment of these regions. Although these studies further suggest that secondary somatosensory cortex and contralateral primary somatosensory cortex are not likely to be responsible for the observed reacquisition in the present study, without a detailed analysis of these regions in our retention lesion animals, their possible involvement in reacquisition of the trace association cannot be excluded.

Our analyses clearly demonstrate that the barrel cortex plays a critical role in learning trace associations when whisker deflection is used as a CS. Furthermore, this study along with our previous analysis of post-training cortical barrel size, strongly suggest that an aspect of the trace association, possibly timing for the $\mathrm{CR}$, either resides in or is regulated by barrel cortex.

\section{Materials and Methods}

\section{Animals}

A total of forty 3-mo-old female New Zealand White albino rabbits (Covance) were used in the present study. All rabbits were housed individually, on a 14-h light/10-h dark cycle, and fed ad libitum. All procedures described were performed in accordance with guidelines approved by Northwestern University's Animal Care and Use Committee.

\section{Surgical procedures}

Rabbits received cortical infusions of ibotenic acid (Lesion) or saline vehicle (Sham), prior to either $10 \mathrm{~d}$ of trace conditioning (trace training; acquisition groups) or five subsequent days of retention testing (trace testing; retention groups) (Fig. 1). The surgical procedures involved were identical except where specifically noted. All surgeries were performed using sterile procedures. Ketamine $(60 \mathrm{mg} / \mathrm{kg})$ and Xylazine $(10 \mathrm{mg} / \mathrm{kg})$ were administered intramuscularly to anesthetize animals prior to surgery. Eyes were kept moist with a thin layer of antibacterial ophthalmic ointment. Animals were positioned in a stereotaxic frame, with lambda $1.5 \mathrm{~mm}$ below bregma. An incision was made along the midline of the scalp, and the scalp and underlying fascia were retracted, exposing the skull. Four holes were drilled in the skull for the placement of self-tapping stainless-steel anchor screws $\left(\# 2 \times 1 / 4^{\prime \prime}\right)$, two of which would serve as ground and reference contacts during the electrophysiological identification of cortical barrel neurons responsive to manual deflection of B-row whiskers (see Electrophysiological Procedures).

For acquisition animals, a 3.0-mm-diameter hole was drilled in the skull overlying the cortical barrel field, centered at $7.0 \mathrm{~mm}$ lateral and $2.0 \mathrm{~mm}$ posterior to bregma, contralateral to the trained side of the face. Barrels with neurons responsive to deflection of B-row whiskers were identified (see Electrophysiological Procedures). The coordinates at which responsive neurons were found were used as the infusion locus of either $3.0 \mu \mathrm{L}$ of ibotenic acid (lesion group) or saline vehicle (sham group) (see Infusion Procedures). Following infusion, the opening in the skull was packed with Gelfoam (Pharmacacia \& Upjohn), and an atraumatic headbolt assembly was attached to the skull. A $1.0-\mathrm{cm}$ length of 26-ga. hypodermic tubing was positioned vertically above bregma, which was fixed in place with dental acrylic. Dental acrylic was used to cover the surface of the skull, as well as to secure the headbolt assembly in place for subsequent behavioral training.

For retention animals, during the initial surgical procedure, a $1.0-\mathrm{cm}$ length of 26-ga. hypodermic tubing, positioned vertically above bregma, and atraumatic headbolt assembly were affixed to the skull surface, but no hole overlaying the barrel cortex was drilled. Following the first auditory test session (see Behavioral Training), animals were prepared for a second surgery as described above. The dental acrylic overlying the barrel cortex was removed, and a 3.0-mm-diameter hole was drilled through the skull, $7.0 \mathrm{~mm}$ lateral and $2.0 \mathrm{~mm}$ posterior to the 26-ga. hypodermic tubing marking bregma. The same procedure for identification of neurons responsive to manual B-row vibrissae deflection and the injection of ibotenic acid or saline vehicle for acquisition lesion and sham animals was used. Following this procedure, the hole in the skull was packed with Gelfoam and covered again with dental acrylic.

Following all surgical procedures, animals were administered buprenorphine $(0.06 \mathrm{mg} / \mathrm{kg})$ at 12 -h intervals for $36 \mathrm{~h}$ to minimize post-surgical discomfort.

\section{Electrophysiological procedure}

While still anesthetized, a pair of stainless-steel screws set into the skull served as ground and reference connections. A single, Teflon-coated, stainless-steel wire electrode $(50-\mu \mathrm{m}$ diameter bare, 112- $\mu \mathrm{m}$ diameter coated, $0.5 \mathrm{M} \Omega$ ) was lowered through a small incision in the dura, at $7.25 \mathrm{~mm}$ lateral and $2.25 \mathrm{~mm}$ posterior to bregma. The electrode was lowered to an initial depth of $0.8 \mathrm{~mm}$ ventral to the dura, where it remained at rest for $5 \mathrm{~min}$ to allow the tissue to settle, and was then lowered at additional $100-\mu \mathrm{m}$ increments. Following each incremental increase in depth, vibrissae on the contralateral side of the head were stimulated manually with a cotton-tipped applicator. Neuronal activity from the electrode was passed to a headstage amplifier (HS-27) via a customized adapter, buffered by the HS-27, filtered (600$6000 \mathrm{~Hz})$, amplified $(20,000 \times)$, and monitored using a Neuralynx Cheetah-32 System (Neuralynx) with an audio channel. If no responsive neurons were identified between depths of 0.8-1.4 $\mathrm{mm}$ ventral to the dura, the same procedure was repeated at adjacent ML/AP coordinates until neurons responsive to B-row vibrissae deflection were identified.

\section{Infusion procedure}

Infusions of $3 \mu \mathrm{L}$ of either $1.0 \%$ ibotenic acid or saline vehicle were made at the same coordinates at which neurons responsive to B-row vibrissae deflection were monitored. Infusions were made using a $10-\mu \mathrm{L}$ Hamilton syringe with a 26 -ga. hypodermic needle. The needle was lowered to a depth of $0.9 \mathrm{~mm}$ ventral to the dura, where it remained at rest for $5 \mathrm{~min}$ to allow the tissue to settle. The $3 \mu \mathrm{L}$ volume was injected in 100-nL increments, separated by 1-min intervals. After the full volume was injected, the syringe was maintained at depth for an additional $5 \mathrm{~min}$ and then removed.

\section{Behavioral training}

Training for all animals began $7 \mathrm{~d}$ following the initial surgical procedure (Fig. 1). During habituation and conditioning sessions, rabbits were restrained up to the neck using a cloth bag and Plexiglas restrainer, from which the head was allowed to protrude. The lids of the trained eye were held open with a Velcro strap and two stainless steel dress hooks. A lightweight aluminum assembly secured an infrared sensor and air-puff delivery tube to the headbolt for the duration of each training session. The head was immobilized by an adjustable bar fixed to the headbolt.

Computers running routines written on LabView software controlled the delivery of all stimuli and acquired behavioral data. Each trace conditioning session consisted of 80 trials, with 
a pseudo-randomly varied intertrial interval (ITI) of 30-60 sec. A 1750-msec behavioral data window was recorded for each trial, consisting of a 500-msec pre-CS baseline interval, 250-msec CS and 150 -msec US intervals separated by a $500-\mathrm{msec}$ stimulus-free trace interval, and a 350-msec post-US interval. For the CS, whiskers B1-B6 were attached $1.0-1.5 \mathrm{~cm}$ from the animal's face to a stock card paper applicator that was then attached to a flat piezoceramic strip (T220-A4-303; Piezo Systems). To generate the CS, 120-V AC was delivered to the piezoceramic strip generating a $60-\mathrm{Hz}, 130-\mu \mathrm{m}$ dorsal-ventral deflection of the attached whiskers (Das et al. 2001; Galvez et al. 2006). All efforts were made to insure that the piezoceramic strip did not contact any whiskers in adjacent rows. White noise $(70 \mathrm{~dB})$ was also generated from speakers on each side of the animal's head throughout all habituation and conditioning sessions to reduce the likelihood of auditory stimuli contributing to CR performance. An air-puff (3.5 psi) to the eye ipsilateral to the trained whiskers served as the US. The infrared sensor suspended in front of the eye measured reflectance from the surface of the cornea. The computer recorded extension of the nictitating membrane across the surface of the cornea as an increase in signal voltage. This voltage was recorded by the computer at $1 \mathrm{kHz}$ for a total of 1750 data points per trial. An eyeblink was defined on a trial-by-trial basis, as an increase in voltage $>4$ SDs above the mean baseline voltage. Conditioned eyeblinks that persisted through US onset were defined as "adaptive" CRs. Pseudo-conditioned animals received 80 presentations of the CS and 80 presentations of the US, pseudo-randomly presented in an unpaired fashion (pseudo-randomly varied 15-30 sec ITI). Trace and pseudo-conditioning sessions were both $1 \mathrm{~h}$ in duration.

Following $1 \mathrm{~d}$ of habituation, all animals received 10 daily sessions of either trace or pseudo-conditioning. On the day following the 10th day of trace training, all rabbits received the first of two 20 trial auditory tests. The auditory tests were identical to the trace training sessions, with the exception that the piezoceramic stimulator was not physically attached, but adjacent, to the trained whiskers. These abbreviated sessions were designed to test for the possibility of an auditory stimulus contributing to CR performance.

Immediately following the first auditory test, the second surgery was performed on those animals assigned to the retention group, to identify and then infuse either saline or ibotenic acid into B-row cortical barrels. Following a $7 \mathrm{~d}$ post-operative recovery period, all animals were trained for an additional $5 \mathrm{~d}$ to test the effect of ibotenic acid lesions on the retention of the previously acquired conditioned response. On the day following the fifth trace testing session, the second auditory test was performed.

Finally, all trace and pseudo-conditioned animals underwent $4 \mathrm{~d}$ of delay conditioning. During delay conditioning, the 500-msec trace interval was decreased to $0 \mathrm{msec}$, so the whisker deflection CS immediately preceded the $150-\mathrm{msec}$ air-puff US. Criterion performance during delay conditioning was a single session performance with a minimum of $80 \%$ adaptive CRs within a block of 10 consecutive trails.

\section{Histology}

Animals were euthanized by intravenous administration of a lethal dose of sodium pentobarbital $(97.5 \mathrm{mg} / \mathrm{kg})$. All animals were then perfused transcardially with $1.0 \mathrm{~L}$ of phosphate buffered saline (PBS; pH 7.4) followed by $1.0 \mathrm{~L} 2 \%$ paraformaldehyde for light fixation. The neocortex was dissected off, flattened (Strominger and Woolsey 1987) and post-fixed overnight in 4\% paraformaldehyde. Flattened cortices were then cryoprotected in 30\% sucrose in PBS and sectioned $(30 \mu \mathrm{m})$ parallel to the cortical surface with a cryostat.

For barrel and lesion visualization, every second and third section was stained for either CO or Nissl, as previously described (Galvez et al. 2006). Briefly, for CO free-floating sections were placed in staining solution $(0.05 \% \mathrm{DAB}, 0.03 \%$ cytochrome $\mathrm{C}$, $4 \%$ sucrose in PBS) for $6 \mathrm{~h}$ at $37^{\circ} \mathrm{C}$ to ensure consistent staining. Staining for CO was then terminated via a series of PBS washes, and sections were then mounted onto gelatin-coated slides for histological analysis of cortical whisker barrels. For Nissl staining, sections were mounted onto slides and stained according to a standard cresyl violet staining protocol.

\section{Analysis}

Four animals were dropped from the experiment due to health concerns, leaving a total of 36 animals. Statistical analyses were conducted on seven acquisition sham, six acquisition lesion, three acquisition pseudo sham, four acquisition pseudo lesion, eight retention sham, and eight retention lesion animals using a mixed general-linear-model (GLM)-ANOVA on Statistical Analysis Software (SAS; SPSS Science) with days of training as withinanimal and training group as between-animal variables. In specific instances, post hoc analyses were performed to determine individual group and day differences.

\section{Acknowledgments}

This work was supported by Mechanisms of Aging and Dementia Training Program AG20506, R01-MH47340, and SFN-MNFP MH20069.

\section{References}

Berger, T.W. and Bassett, J.L. 1992. System properties of the hippocampus. In Learning and memory: The behavioral and biological substrates. (eds. I. Gormezano and E.A. Wasserman). Lawrence Erlbaum Assoc., Inc., Hillsdale, NJ.

Catalano, S.M., Robertson, R.T., and Killackey, H.P. 1995. Rapid alteration of thalamocortical axon morphology follows peripheral damage in the neonatal rat. Proc. Natl. Acad. Sci. 92: 2549-2552.

Clark, R.E. and Squire, L.R. 1998. Classical conditioning and brain systems: The role of awareness. Science 280: 77-81.

Clark, G.A., McCormick, D.A., Lavond, D.G., and Thompson, R.F. 1984. Effects of lesions of cerebellar nuclei on conditioned behavioral and hippocampal neuronal responses. Brain Res. 291: 125-136.

Darian-Smith, C. and Gilbert, C.D. 1994. Axonal sprouting accompanies functional reorganization in adult cat striate cortex. Nature 368: 737-740.

Darian-Smith, C. and Gilbert, C.D. 1995. Topographic reorganization in the striate cortex of the adult cat and monkey is cortically mediated. I. Neurosci. 15: 1631-1647.

Das, S., Weiss, C., and Disterhoft, J.F. 2001. Eyeblink conditioning in the rabbit (Oryctolagus cuniculus) with stimulation of the mystacial vibrissae as a conditioned stimulus. Behav. Neurosci. 115: 731-736.

Diamond, D.M. and Weinberger, N.M. 1986. Classical conditioning rapidly induces specific changes in frequency receptive fields of single neurons in secondary and ventral ectosylvian auditory cortical fields. Brain Res. 372: 357-360.

Diamond, D.M. and Weinberger, N.M. 1989. Role of context in the expression of learning-induced plasticity of single neurons in auditory cortex. Behav. Neurosci. 103: 471-494.

Disterhoft, J.F. and Stuart, D.K. 1976. Trial sequence of changed unit activity in auditory system of alert rat during conditioned response acquisition and extinction. J. Neurophysiol. 39: 266-281.

Edeline, J.M., Pham, P., and Weinberger, N.M. 1993. Rapid development of learning-induced receptive field plasticity in the auditory cortex. Behav. Neurosci. 107: 539-551.

Eichenbaum, H., Otto, T., and Cohen, N.J. 1992. The hippocampus-What does it do? Behav. Neural Biol. 57: 2-36.

Frankland, P.W., Ding, H.K., Takahashi, E., Suzuki, A., Kida, S., and Silva, A.J. 2006. Stability of recent and remote contextual fear memory. Learn. Mem. 13: 451-457.

Galvez, R., Weiss, C., Weible, A.P., and Disterhoft, J.F. 2006. Vibrissa-signaled eyeblink conditioning induces somatosensory cortical plasticity. J. Neurosci. 26: 6062-6068.

Ghose, G.M., Yang, T., and Maunsell, J.H. 2002. Physiological correlates of perceptual learning in monkey V1 and V2. J. Neurophysiol. 87: 1867-1888.

Han, C.J., O'Tuathaigh, C.M., Trigt, L., Quinn, J.J., Fanselow, M.S., Mongeau, R., Koch, C., and Anderson, D.J. 2003. Trace but not delay fear conditioning requires attention and the anterior cingulate cortex. Proc. Natl. Acad. Sci. 100: 13087-13092.

Hurwitz, B.E., Dietrich, W.D., McCabe, P.M., Watson, B.D., Ginsberg, M.D., and Schneiderman, N. 1990. Sensory-motor deficit and recovery from thrombotic infarction of the vibrissal barrel-field cortex. Brain Res. 512: 210-220.

Hurwitz, B.E., Dietrich, W.D., McCabe, P.M., Alonso, O., Watson, B.D. Ginsberg, M.D., and Schneiderman, N. 1991. Amphetamine 
promotes recovery from sensory-motor integration deficit after thrombotic infarction of the primary somatosensory rat cortex. Stroke 22: 648-654.

Jenkins, W.M., Merzenich, M.M., Ochs, M.T., Allard, T., and Guic-Robles, E. 1990. Functional reorganization of primary somatosensory cortex in adult owl monkeys after behaviorally controlled tactile stimulation. J. Neurophysiol. 63: 82-104.

Jensen, K.F. and Killackey, H.P. 1987. Terminal arbors of axons projecting to the somatosensory cortex of the adult rat. II. The altered morphology of thalamocortical afferents following neonatal infraorbital nerve cut. J. Neurosci. 7: 3544-3553.

Kim, J.J., Clark, R.E., and Thompson, R.F. 1995. Hippocampectomy impairs the memory of recently, but not remotely, acquired trace eyeblink conditioned responses. Behav. Neurosci. 109: 195-203.

Kitzes, L.M., Farley, G.R., and Starr, A. 1978. Modulation of auditory cortex unit activity during the performance of a conditioned response. Exp. Neurol. 62: 678-697.

Kraus, N. and Disterhoft, J.F. 1981. Location of rabbit auditory cortex and description of single unit activity. Brain Res. 214: 275-286.

Kronforst-Collins, M.A. and Disterhoft, J.F. 1998. Lesions of the caudal area of rabbit medial prefrontal cortex impair trace eyeblink conditioning. Neurobiol. Learn. Mem. 69: 147-162.

Krupa, D.J., Ghazanfar, A.A., and Nicolelis, M.A. 1999. Immediate thalamic sensory plasticity depends on corticothalamic feedback. Proc. Natl. Acad. Sci. 96: 8200-8205.

Krupa, D.J., Wiest, M.C., Shuler, M.G., Laubach, M., and Nicolelis, M.A 2004. Layer-specific somatosensory cortical activation during active tactile discrimination. Science 304: 1989-1992.

Leal-Campanario, R., Delgado-Garcia, J.M., and Gruart, A. 2006. Microstimulation of the somatosensory cortex can substitute for vibrissa stimulation during Pavlovian conditioning. Proc. Natl. Acad. Sci. 103: 10052-10057.

Mauk, M.D. and Thompson, R.F. 1987. Retention of classically conditioned eyelid responses following acute decerebration. Brain Res. 403: 89-95.

McEchron, M.D. and Disterhoft, J.F. 1997. Sequence of single neuron changes in CA1 hippocampus of rabbits during acquisition of trace eyeblink conditioned responses. J. Neurophysiol. 78: 1030-1044.

McEchron, M.D., Weible, A.P., and Disterhoft, J.F. 2001. Aging and learning-specific changes in single-neuron activity in CA1 hippocampus during rabbit trace eyeblink conditioning. J. Neurophysiol. 86: 1839-1857.

McGlinchey-Berroth, R., Carrillo, M.C., Gabrieli, J.D., Brawn, C.M., and Disterhoft, J.F. 1997. Impaired trace eyeblink conditioning in bilateral, medial-temporal lobe amnesia. Behav. Neurosci. 111: 873882.

McKenna, J.T. and Vertes, R.P. 2004. Afferent projections to nucleus reuniens of the thalamus. J. Comp. Neurol. 480: 115-142.

McLaughlin, J., Skaggs, H., Churchwell, J., and Powell, D.A. 2002. Medial prefrontal cortex and Pavlovian conditioning: Trace versus delay conditioning. Behav. Neurosci. 116: 37-47.

Moore, J.W., Yeo, C.H., Oakley, D.A., and Russell, I.S. 1980. Conditioned inhibition of the nictitating membrane response in decorticate rabbits. Behav. Brain Res. 1: 397-409.

Moyer Jr., J.R., Deyo, R.A., and Disterhoft, J.F. 1990. Hippocampectomy disrupts trace eye-blink conditioning in rabbits. Behav. Neurosci. 104: $243-252$.

Norman, R.J., Buchwald, J.S., and Villablanca, J.R. 1977. Classical conditioning with auditory discrimination of the eye blink in decerebrate cats. Science 196: 551-553.

Oakley, D.A. and Russell, I.S. 1972. Neocortical lesions and Pavlovian conditioning. Physiol. Behav. 8: 915-926.

Oakley, D.A. and Russell, I.S. 1975. Role of cortex in Pavlovian discrimination learning. Physiol. Behav. 15: 315-321.

Oakley, D.A. and Russell, I.S. 1977. Subcortical storage of Pavlovian conditioning in the rabbit. Physiol. Behav. 18: 931-937.

Pazos, A.J., Orezzoli, S.L., McCabe, P.M., Dietrich, W.D., and Green, E.J. 1995. Recovery of vibrissae-dependent behavioral responses following barrelfield damage is not dependent upon the remaining somatosensory cortical tissue. Brain Res. 689: 224-232.

Powell, D.A. and Churchwell, J. 2002. Mediodorsal thalamic lesions impair trace eyeblink conditioning in the rabbit. Learn. Mem. 9: 10-17.

Recanzone, G.H., Merzenich, M.M., Jenkins, W.M., Grajski, K.A., and Dinse, H.R. 1992. Topographic reorganization of the hand representation in cortical area $3 \mathrm{~b}$ owl monkeys trained in a frequency-discrimination task. J. Neurophysiol. 67: 1031-1056.

Recanzone, G.H., Schreiner, C.E., and Merzenich, M.M. 1993. Plasticity in the frequency representation of primary auditory cortex following discrimination training in adult owl monkeys. J. Neurosci. 13: $87-103$.

Rutkowski, R.G. and Weinberger, N.M. 2005. Encoding of learned importance of sound by magnitude of representational area in primary auditory cortex. Proc. Natl. Acad. Sci. 102: 13664-13669.

Schoups, A., Vogels, R., Qian, N., and Orban, G. 2001. Practising orientation identification improves orientation coding in V1 neurons. Nature 412: 549-553.

Solomon, P.R., Vander Schaaf, E.R., Thompson, R.F., and Weisz, D.J. 1986. Hippocampus and trace conditioning of the rabbit's classically conditioned nictitating membrane response. Behav. Neurosci. 100: 729-744.

Squire, L.R., Stark, C.E., and Clark, R.E. 2004. The medial temporal lobe. Annu. Rev. Neurosci. 27: 279-306.

Strominger, R.N. and Woolsey, T.A. 1987. Templates for locating the whisker area in fresh flattened mouse and rat cortex. J. Neurosci. Methods 22: 113-118.

Takehara, K., Kawahara, S., Takatsuki, K., and Kirino, Y. 2002. Time-limited role of the hippocampus in the memory for trace eyeblink conditioning in mice. Brain Res. 951: 183-190.

Takehara, K., Kawahara, S., and Kirino, Y. 2003. Time-dependent reorganization of the brain components underlying memory retention in trace eyeblink conditioning. J. Neurosci. 23: 9897-9905.

Theios, J. and Brelsford Jr., J.W. 1966. A Markov model for classical conditioning: Application to eye-blink conditioning in rabbits. Psychol. Rev. 73: 393-408.

Tseng, W., Guan, R., Disterhoft, J.F., and Weiss, C. 2004. Trace eyeblink conditioning is hippocampally dependent in mice. Hippocampus 14: $58-65$.

Van der Werf, Y.D., Witter, M.P., and Groenewegen, H.J. 2002. The intralaminar and midline nuclei of the thalamus. Anatomical and functional evidence for participation in processes of arousal and awareness. Brain Res. Rev. 39: 107-140.

Weible, A.P., McEchron, M.D., and Disterhoft, J.F. 2000. Cortical involvement in acquisition and extinction of trace eyeblink conditioning. Behav. Neurosci. 114: 1058-1067.

Weible, A.P., O'Reilly, J.A., Weiss, C., and Disterhoft, J.F. 2006. Comparisons of dorsal and ventral hippocampus cornu ammonis region 1 pyramidal neuron activity during trace eye-blink conditioning in the rabbit. Neuroscience 141: 1123-1137.

Weiss, C. and Disterhoft, J.F. 1996. Eyeblink conditioning, motor control, and the analysis of limbic-cerebellar interactions. Behav. Brain Sci. 19: 479-481.

Weiss, C., Bouwmeester, H., Power, J.M., and Disterhoft, J.F. 1999. Hippocampal lesions prevent trace eyeblink conditioning in the freely moving rat. Behav. Brain Res. 99: 123-132.

Weiss, C., Weible, A.P., Galvez, R., and Disterhoft, J.F. 2006. Forebrain-cerebellar interactions during learning. Cell Sci. Rev. 3: $200-230$.

Woolsey, T.A. and Van der Loos, H. 1970. The structural organization of layer IV in the somatosensory region (SI) of mouse cerebral cortex. The description of a cortical field composed of discrete cytoarchitectonic units. Brain Res. 17: 205-242.

Received August 30, 2006; accepted in revised form October 31, 2006. 


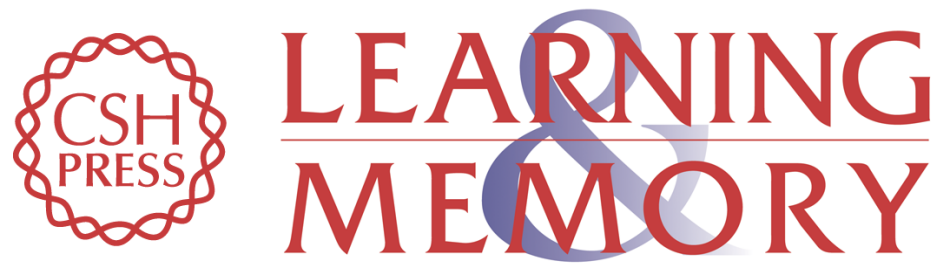

\section{Cortical barrel lesions impair whisker-CS trace eyeblink conditioning}

Roberto Galvez, Aldis P. Weible and John F. Disterhoft

Learn. Mem. 2007, 14:

Access the most recent version at doi:10.1101//m.418407

References This article cites 58 articles, 16 of which can be accessed free at: http://learnmem.cshlp.org/content/14/1-2/94.full.html\#ref-list-1

License

Email Alerting Receive free email alerts when new articles cite this article - sign up in the box at the Service top right corner of the article or click here. 